\title{
LIE GROUPS ISOMORPHIC TO DIRECT PRODUCTS OF UNITARY GROUPS
}

\author{
IVAN VIDAV AND PETER LEGIŠA
}

\begin{abstract}
A criterion is given for a compact connected subgroup of $\mathrm{Gl}(n, \mathrm{C})$ to be isomorphic to a direct product of unitary groups. It implies that a compact connected subgroup of rank $n$ in $\operatorname{Gl}(n, \mathrm{C})$ is isomorphic to a direct product of unitary groups.
\end{abstract}

The paper gives a generalization of some of the results in [3]. Let $G$ be a compact connected subgroup of $\mathrm{Gl}(n, \mathbf{C})$. We denote by $L(G)$ the Lie algebra of $G$ and set $H(G)=i L(G)$. The rank of $G$ is the dimension of a maximal torus in $G$ (see [1, p. 93]).

THEOREM. Let $G$ be a compact connected subgroup of rank $k$ in $\mathrm{Gl}(n, \mathbf{C})$. Suppose there exist $r \geqslant k$ orthogonal idempotents $a_{1}, \ldots, a_{r}$ in $H(G)$. Then $r=$ $k$ and $G$ is isomorphic (as a Lie group) to a direct product of unitary groups: $G \cong U\left(n_{1}\right) \times \cdots \times U\left(n_{m}\right)$ with $n_{1}+\cdots+n_{m}=k$.

Proof. By [2, p. 176, Theorem 1] $G$ is similar to a subgroup of $U(n)$. Hence we may assume that $G$ is a subgroup of $U(n)$. Thus the operators in $H(G) \subset \operatorname{End}\left(\mathbf{C}^{n}\right)$ are hermitian. Since $a_{1}, \ldots, a_{r}$ commute we see that $T=\left\{\exp \left(i t_{1} a_{1}+\cdots+i t_{r} a_{r}\right) \mid t_{1}, \ldots, t_{r} \in \mathbf{R}\right\}$ is a torus in $G$ of dimension $r$. Clearly $r=k$ and $T$ is a maximal torus. If $a \in H(G)$ then $\exp ($ ita $) \in G$ $(t \in \mathbf{R})$ and is contained in some conjugate of $T$ (see [1, p. 89]), i.e. $\exp (i t a) \in u^{-1} T u=u^{*} T u$ for some $u \in G$. It follows that $a=t_{1} u^{*} a_{1} u$ $+\cdots+t_{r} u^{*} a_{r} u$. Since $a^{2}=t_{1}^{2} u^{*} a_{1} u+\cdots+t_{r}^{2} u^{*} a_{r} u$ and $u^{*} a_{s} u \in H(G)$ for $s=1, \ldots, r$ we see that $a^{2} \in H(G)$. Let $b \in H(G)$, too. Since $a b+b a$ $=(a+b)^{2}-a^{2}-b^{2}$ we see that $a b+b a \in H(G)$. Also, $a b-b a \in i H(G)$ since $i a, i b \in L(G)$. Thus $a b \in H(G)+i H(G)$. Let $A(G)=H(G)$ $+i H(G)$. It follows that $A(G)$ is an algebra. Clearly, it is a finite dimensional $C^{*}$-algebra. By the Wedderburn decomposition there exist central idempotents $e_{1}, \ldots, e_{m} \in A(G)=A$ such that $A=A e_{1} \oplus \cdots \oplus A e_{m}$ and $A e_{s}$ is isomorphic to $\operatorname{End}\left(X_{s}\right)$ for some finite dimensional vector space $X_{s}$ over C $(s=1, \ldots, m)$.

The ideal $A e_{s}$ is closed, hence selfadjoint and a $C^{*}$-subalgebra of $A$. Clearly, $e_{s}$ is the identity on $A e_{s}$ and hence $e_{s}^{*}=e_{s}$. Consider the group $V$ of unitary elements in $A e_{s}$. The isomorphism $A e_{s} \cong \operatorname{End}\left(X_{s}\right)$ defines a (continuous) representation of $V$ on $X_{s}$. Using once more [2, p. 176, Theorem 1] we equip $X_{s}$ with an inner product such that the isomorphism maps $V$ into the unitary

Received by the editors May 26, 1975.

AMS (MOS) subject classifications (1970). Primary 22E15, 22E60; Secondary 46L05, 46L20. 
group of $\mathcal{L}\left(X_{s}\right)$, the $C^{*}$-algebra of all linear operators on the Hilbert space $X_{s}$. Consequently, hermitian elements in $A e_{s}$ are mapped into hermitian operators and our isomorphism in an isometric*-isomorphism. We identify the algebras $A e_{s}$ and $\mathfrak{L}\left(X_{s}\right)$ in this sense.

Since exp: $L(G) \rightarrow G$ is surjective, $G \subset A$. If $u \in G$ then $\left(u e_{s}\right)^{*} u e_{s}$ $=e_{s} u^{*} u e_{s}=e_{s}$. Thus $u e_{s}$ is a unitary operator on $X_{s}$. Consider the smooth homomorphism $G \rightarrow U\left(X_{1}\right) \times \cdots \times U\left(X_{m}\right)$ given by $u \mapsto\left(u e_{1}, \ldots, u e_{m}\right)$ $\left(U\left(X_{s}\right)\right.$ denotes the unitary group on $\left.X_{s}\right)$. We claim this homomorphism is onto. Let $u_{1} \in U\left(X_{1}\right)$. There exists a hermitian element $h_{1} \in A e_{1}$ such that $\exp \left(i h_{1}\right)=u_{1}$. Consider $h_{1}$ as an element in $A$. Then $\exp \left(i h_{1}\right)=\left(u_{1}, 1, \ldots, 1\right)$. Observe that the inverse $\left(u e_{1}, \ldots, u e_{m}\right) \mapsto u e_{1}+\cdots+u e_{m}$ is also smooth and that $\operatorname{rank}\left(U\left(n_{1}\right) \times \cdots \times U\left(n_{m}\right)\right)=n_{1}+\cdots+n_{m}$.

Corollary. Let $G$ be a compact connected subgroup of rank $n$ in $\mathrm{Gl}(n, \mathrm{C})$. Then $G$ is isomorphic (as a Lie group) to a direct product of unitary groups.

Proof. As before, we may assume that $G \leqslant U(n)$. Let $T$ be a maximal torus in $G$. Then $i L(T)$ contains $n$ commuting linearly independent hermitian operators, say $h_{1}, \ldots, h_{n}$. It is well known that these operators have a common orthogonal eigenbasis. Thus there exist $s \leqslant n$ orthogonal projections $p_{1}, \ldots, p_{s}$ such that every $h_{i}$ is a linear combination of $p_{1}, \ldots, p_{s}$. Since $h_{1}, \ldots, h_{n}$ are linearly independent, $s=n$. Thus $i L(G)$ contains $n$ orthogonal idempotents and we may use the Theorem.

\section{REFERENCES}

1. J. F. Adams, Lectures on Lie groups, Benjamin, New York and Amsterdam, 1969. MR 40 \#5780.

2. C. Chevalley, Theory of Lie groups. Vol. 1, Princeton Math. Ser., vol. 8, Princeton Univ. Press, Princeton, N.J., 1946. MR 7, 412.

3. I. Vidav, The group of isometries and the structure of a finite dimensional Banach space, Linear Algebra and Appl. (to appear).

Department of Mathematics, University of Lubljana, Ljubljana, Yugoslavia 